\title{
Activités antioxydante et insecticide d'huiles essentielles de Mentha arvensis L. du Sénégal
}

\author{
Serigne Mbacké DIOP ${ }^{1,2^{*}}$, Momar Talla GUEYE ${ }^{1}$, El Hadji Barka NDIAYE ${ }^{1,2}$, \\ Abdoulaye THIAM ${ }^{1,2}$, Papa Seyni CISSOKHO ${ }^{1}$, Cheikhna Hamala SANGHARE ${ }^{1,2}$ \\ et Marie-Laure FAUCONNIER ${ }^{3}$
}

\author{
${ }^{1}$ Laboratoire des Analyses Phytosanitaires, Institut de Technologie Alimentaire, BP 2765, \\ Hann-Dakar, Sénégal. \\ ${ }^{2}$ Département de Chimie, Faculté des Sciences et Techniques, Université Cheikh Anta Diop, BP 5005, \\ Dakar, Sénégal. \\ ${ }^{3}$ Chimie Générale et Organique, Département Agro-Bio-Chem, Gembloux Agro-Bio Tech, Université de \\ Liège 2, Passage des Déportés-5030, Gembloux, Belgique. \\ *Auteur correspondant ; E-mail : serigneami@ hotmail.fr ; Tél. : 00221779738173.
}

\section{REMERCIEMENTS}

Au terme de cette étude, nous tenons à remercier la Wallonie Bruxelles Internationale (WBI) qui a financé le projet «WBI-Sénégal $n^{\circ} 2:$ Production d'huiles essentielles à partir de plantes locales: expérimentation, adaptation et diffusion de technologies » qui nous a permis d'effectuer ce travail.

\begin{tabular}{lll}
\hline Received: 27-04-2021 & Accepted: 15-06-2021 & Published: 30-06-2021 \\
\hline
\end{tabular}

\section{RESUME}

Ce travail s'inscrit dans le contexte global de valorisation de la biodiversité des plantes aromatiques du Sénégal. L'objectif de l'étude était de déterminer la composition chimique d'huiles essentielles (HE) de Mentha arvensis L. et d'évaluer leurs activités antioxydante et insecticide. A cet effet, des feuilles de $M$. arvensis ont été récoltées dans la région de Dakar (Sénégal) et séchées à l'ombre puis leurs HE extraites par entrainement à la vapeur d'eau. Les HE obtenues ont été analysées par chromatographie en phase gazeuse (CPG) couplée à un détecteur à ionisation de flamme (FID) et un spectromètre de masse (MS). L'activité antioxydante des HE a été évaluée par la méthode d'inhibition du radical 2,2-diphényl-1-picrylhydrazyl (DPPH) tandis que l'activité insecticide a été testée par fumigation contre Sitophilus zeamais (Motsch.). Les résultats ont montré que les HE contiennent principalement du menthol $(68,4 \%)$, de l'acétate de menthyle $(16,9 \%)$ et de la menthone $(5,0 \%)$. Leur activité antioxydante augmente avec la concentration, soient $38 \%, 57 \%$ et $73 \%$ d'inhibition respectivement pour 10,25 et $50 \mathrm{mg} / \mathrm{ml}$; la $\mathrm{CI}_{50}$ est de $19,5 \mathrm{mg} / \mathrm{ml}$. Les huiles présentent aussi une activité insecticide. Cette dernière bien qu'étant faible après 24 heures, augmente fortement au bout de 48 heures avec une mortalité maximale de $92 \%$. Cette mortalité atteint des maximums de $98 \%$ pour 5 et $25 \mu 1$ et $100 \%$ pour 50 et $75 \mu 1$ après 72 heures.

(C) 2021 International Formulae Group. All rights reserved.

Mots clés : Mentha arvensis L., huiles essentielles, menthol, activité antioxydante, activité insecticide. 


\title{
Antioxidant and insecticidal activities of essential oils of Mentha arvensis $\mathbf{L}$. from Senegal
}

\begin{abstract}
This work is part of the global context of valorization of the biodiversity of aromatic plants from Senegal. The aim of the study is to determine the chemical composition of essential oils (EOs) of Mentha arvensis L. and to evaluate their antioxidant and insecticidal activities. For this purpose, leaves of $M$. arvensis were harvested in Dakar region, Senegal and dried in the shade. EOs were extracted by steam distillation from dried leaves and analyzed by gas chromatography (GC) coupled to a flame ionization detector (FID) and a mass spectrometer (MS). The antioxidant activity of EOs was assessed using the 2,2-diphenyl-1-picrylhydrazyl (DPPH) method while the insecticidal activity was tested by fumigation against Sitophilus zeamais (Motsch.). The results revealed that EOs were dominated by menthol (68.4\%), menthyl acetate $(16.9 \%)$ and menthone $(5.0 \%)$. The antioxidant activity assay showed that the inhibition increased with the concentration: 38\%, 57\% and 73\% for 10, 25 and 50 $\mathrm{mg} / \mathrm{ml}$, respectively; the $\mathrm{IC}_{50}$ was of $19.5 \mathrm{mg} / \mathrm{ml}$. The oils were also effective as insecticide. Mortalities were low after 24 hours and increased strongly after 48 hours with a maximum of $92 \%$. They reached a maximum of $98 \%$ for 5 and $25 \mu \mathrm{l}$ and $100 \%$ for 50 and $75 \mu 1$ after 72 hours.
\end{abstract}

(C) 2021 International Formulae Group. All rights reserved.

Keywords: Mentha arvensis L., essential oils, menthol, antioxydant activity, insecticidal activity.

\section{INTRODUCTION}

En Afrique subsaharienne, les plantes aromatiques et à parfum représentent un grand intérêt pour les populations. Au Sénégal, les plantes à huiles essentielles (HE) sont traditionnellement utilisées par les populations locales pour l'aromatisation des boissons et tisanes, les arts culinaires, leurs vertus médicinales, la parfumerie et même à des fins mystiques (Diop, 2018). Cette utilisation traditionnelle résulte souvent de connaissances ancestrales accumulées dans le temps, puis transmises de génération en génération grâce à la tradition orale.

La flore sénégalaise est très riche en plantes aromatiques. Entre autres plantes de cette flore, nous pouvons citer Citrus sinensis, Cybopogon citratus, Eucalyptus camaldulensis, Hyptis suaveolens, Mentha $x$ piperita et Ocimum basilicum. La menthe est l'une des plantes aromatiques les plus cultivées au Sénégal. Les espèces couramment rencontrées sont Mentha aquatica L., M. arvensis L., M. longifolia L., M. x piperita L., et $M$. spicata L. Toutefois, il faut signaler qu'une bonne partie de la production est consommée à l'état brut. Pourtant si ces plantes étaient bien étudiées et les substances qu'elles contiennent caractérisées, il serait possible de les valoriser autrement, notamment dans la protection des denrées stockées, la conservation des aliments, la phytopharmacie, etc. (Khani et Asghari, 2012; Guèye et al., 2013 ; Ngom et al., 2014; Cissokho et al., 2015; Ngom et al., 2018).

Le domaine d'application d'une huile essentielle dépend de sa composition chimique. Cette dernière donne les principaux constituants de l'huile qui sont souvent responsables de ses propriétés. La littérature montre plusieurs études réalisées sur les HE de menthes (Verma et al., 2010; Curutchet et al., 2014; Dai et al., 2015; Diop et al., 2016). Mentha arvensis est l'une des espèces les plus importantes du genre Mentha. Les études rapportées sur les HE de cette espèce montrent de fortes teneurs de menthol : 73,0\% (Rao, 1999), 61,9-82,2\% (Verma et al., 2010) et 73,7$85,8 \%$ (Padalia et al., 2013).

Les huiles essentielles possèdent d'importantes propriétés biologiques: antibactériennes, anti-inflammatoires, antioxydantes, insecticides, etc. (Boukhebti et al., 2011; El-hawary et al., 2013; Ncibi et al., 2020; Thiam et al., 2020). D'ailleurs, plusieurs études ont montré que cette activité antioxydante est liée à la présence de dérivés phénoliques (Nguyen et al., 2010 ; Kwee et 
Niemeyer, 2011). Les plantes à HE sont aussi couramment utilisées comme insecticides (Guèye et al., 2011; Cissokho et al., 2015). En Afrique, les insectes ravageurs parmi lesquels Callosobruchus maculatus Fab., Caryedon serratus Olivier, Prostephanus truncatus Horn, Sitophilus zeamais Motsch. et Tribolium castaneum Herbst causent d'énormes pertes sur les denrées stockées. Pour lutter contre ces ravageurs, les agriculteurs ont recours aux pesticides de synthèse. Ces derniers, bien qu'efficaces, causent d'énormes dégâts sur l'environnement et la santé (Cissokho et al., 2015). Il est alors souhaitable de disposer d'alternatives efficaces et plus respectueuses de l'environnement. A cet effet, il est rapporté l'efficacité d'HE contre les insectes ravageurs de céréales et légumineuses en stockage (Ilboudo et al., 2010; Cardiet et al., 2012; Ngom et al., 2018).

Le présent travail s'inscrit dans le contexte de valorisation de la biodiversité des plantes aromatiques du Sénégal par la recherche de bio-substances actives, en partie substituables aux pesticides de synthèse. Il a pour objectif de déterminer la composition chimique d'huiles essentielles de $M$. arvensis et d'évaluer leur efficacité comme antioxydant et insecticide.

\section{MATERIEL ET METHODES Matériel biologique La menthe}

Les feuilles de Mentha arvensis L. utilisées ont été récoltées à Dakar. Les feuilles ont été, par la suite, séchées au laboratoire à température ambiante pendant une durée de 7 jours. Leur identification a été effectuée à l'Institut Fondamental d'Afrique Noire (IFAN) de Dakar où un spécimen a été déposé sous le numéro $\mathrm{LB}_{5}$.

\section{Le maïs}

Le maïs (Zea mays L.) utilisé a été acheté au marché Castors de Dakar. Après tri, il a été exposé au soleil pendant une journée puis conservé au réfrigérateur au moins une semaine afin d'enrayer toute infestation insidieuse. Avant leur utilisation, les grains ont été remis à la température ambiante du laboratoire.

\section{Les insectes}

De jeunes adultes de Sitophilus zeamais (Motsch.) de troisième génération ont été utilisés pour les tests. Ces insectes sont issus d'un élevage de masse avec du maïs dans les conditions ambiantes du laboratoire.

\section{Méthodes
Extraction et caractérisation des huiles essentielles

Les HE ont été extraites des feuilles sèches de $M$. arvensis ci-dessus décrites par distillation à l'aide d'un dispositif de type Clevenger pendant $30 \mathrm{~min}$. Les HE obtenues ont été conservées au réfrigérateur $\left(\right.$ à $4{ }^{\circ} \mathrm{C}$ ) dans des flacons ambrés.

L'analyse des HE a été effectuée par chromatographie en phase gazeuse (CPG) couplée à un détecteur à ionisation de flamme (FID) et un spectromètre de masse (MS). A cet effet, des solutions d'huiles essentielles (10 $\mathrm{mg} / 10 \mathrm{ml}, \mathrm{HE} / n$-hexane) ont été préparées et diluées quatre fois avant analyse. Le volume d'échantillon injecté par analyse est de $1 \mu \mathrm{l}$. Les conditions chromatographiques utilisées sont les suivantes : température de l'injecteur (en mode Splitless) $280{ }^{\circ} \mathrm{C}$ et température du détecteur $290{ }^{\circ} \mathrm{C}$; four : température initiale 40 ${ }^{\circ} \mathrm{C}$ (5 min), élévation graduelle de $8{ }^{\circ} \mathrm{C} / \mathrm{min}$ jusqu'à $280^{\circ} \mathrm{C}(5 \mathrm{~min})$; gaz vecteur, hélium à flux constant de $1,5 \mathrm{ml} / \mathrm{min}$; flux d'air et d'hydrogène, respectivement $350 \mathrm{ml} / \mathrm{min}$ et 35 $\mathrm{ml} / \mathrm{min}$. La colonne utilisée est de type Optima5-MS-Accent (Macherey-Nagel, DürenGermany), 5\%-phényl-95\%-méthylsiloxane (30 $\mathrm{m} \times 0,25 \mathrm{~mm} ; 0,25 \mu \mathrm{m}$ épaisseur du film).

CPG/FID - Les pourcentages des constituants des HE ont été déterminés grâce à un chromatographe en phase gazeuse (Trace Ultra GC de Thermo Electron Corporation, Interscience Louvain-La-Neuve, Belgium) couplé à un détecteur à ionisation de flamme. La proportion de chaque composé (exprimée en \%) représente le rapport de l'aire intégrée par ce dernier sur la somme des aires de tous les composés. 
CPG/MS - Un spectromètre de masse (Agilent 5973 Network Mass Selective Detector Quadrupole) couplé à un chromatographe en phase gazeuse (Agilent $6890 \mathrm{~N}(\mathrm{G} 1530 \mathrm{~N})$, USA) a été utilisé pour l'identification des composés. Les spectres de masse ont été obtenus par impact électronique à $70 \mathrm{eV}$ et le poids moléculaire des pics étaient compris entre 50 et $550 \mathrm{amu}$.

L'identification des constituants des HE a été effectuée par comparaison de leur spectre de masse et indice de rétention avec ceux disponibles dans une base de données informatisées (Wiley 275 L) et dans la littérature (Joulain et König, 1998; Adams, 2001). Des composés purs (SIGMA ALDRICH-Boornem, Belgium) ont été aussi injectés dans les mêmes conditions que les HE afin de confirmer la présence des constituants majoritaires.

\section{Mesure de l'activité antioxydante par spectrophotométrie $U$ V-VIS}

L'évaluation de l'activité antioxydante a été réalisée à l'aide du test au 2,2-diphényl-1picrylhydrazyl (DPPH). Ce dernier est mauve lorsqu'il est oxydé (état naturel, composé de radicaux libres stables) et devient jaunetransparent lorsqu'il est réduit (en présence d'antioxydant). Ce test a été réalisé grâce à une mesure ponctuelle de l'absorbance à $517 \mathrm{~nm}$ d'un mélange constitué de $1,5 \mathrm{ml}$ de solution d'HE et $1,5 \mathrm{ml}$ de solution méthanolique de DPPH de concentration $2,5.10^{-2} \mathrm{mg} / \mathrm{ml}$ gardée à l'abri de la chaleur et de la lumière. Les HE ont été testées à différentes concentrations : 10 , 25 et $50 \mathrm{mg} / \mathrm{ml}$ tandis que le témoin positif, trolox a été utilisé aux concentrations de $10^{-3}$;
$5.10^{-3} ; 10^{-2}$ et $10^{-1} \mathrm{mg} / \mathrm{ml}$, le méthanol étant le témoin négatif.

La lecture de l'absorbance a été effectuée par spectrophotométrie UV contre un blanc préparé pour chaque concentration après 30 min d'incubation à l'obscurité et à température ambiante. Chaque analyse a été répétée 3 fois. Les résultats ont été exprimés en pourcentage d'inhibition (I\%) des radicaux libres en utilisant la formule suivante :

$$
I(\%)=\frac{\text { Absorbance contrôle négatif }- \text { Absorbance échantillon }}{\text { Absorbance contrôle négatif }} \times 100
$$

\section{Evaluation de l'activité insecticide}

Les tests ont été conduits par fumigation dans des bocaux en verre d'une capacité de 200 ml. Le dispositif expérimental comporte six (6) traitements. A cet effet, $25 \mathrm{~g}$ de maïs et 20 insectes non sexés ont été placés dans chaque bocal. Les HE ont été déposées sur du coton placé sous le couvercle des bocaux à différentes doses : $5 ; 25 ; 50$ et $75 \mu$ l diluées chacune dans $50 \mu \mathrm{l}$ d'acétone. Chaque traitement a été répété quatre fois afin de permettre le calcul des mortalités moyennes. Un témoin blanc (sans HE ni acétone) et un témoin à l'acétone ont été aussi utilisés (Tableau 1).

Le décomptage des insectes morts a été effectué 24,48 et 72 heures après le début des tests. La formule d'Abbott (1925) a été utilisée pour corriger la mortalité naturelle selon la formule suivante :

$$
\begin{aligned}
& \text { Mcorr }=\left(\frac{M t-M c}{100-M c}\right) \times 100 \\
& \text { Mcorr }=\text { mortalité corrigée }(\%) \\
& M t=\text { Mortalité dans le groupe traité } \\
& \text { Mc }=\text { Mortalité dans le groupe témoin }
\end{aligned}
$$

Tableau 1 : Liste des traitements.

$\begin{array}{lcccccc}\text { Nom des traitements } & \text { T0 } & \text { T1 } & \text { T2 } & \text { T3 } & \text { T4 } & \text { T5 } \\ \text { Concentration (HE/acétone ; v/v) } & 00 / 00 & 00 / 50 & 05 / 50 & 25 / 50 & 50 / 50 & 75 / 50\end{array}$




\section{RESULTATS}

\section{Composition chimique}

L'extraction a donné un rendement de $0,19 \%$ en huiles essentielles. L'analyse chromatographique a permis d'identifier 20 composés dans les HE de $M$. arvensis. Ces dernières sont principalement dominées par les monoterpènes qui constituent les $98,6 \%$ alors que les sesquiterpènes n'en représentent que $1,4 \%$ (Tableau 2). Les principaux composés obtenus dans les HE sont le menthol $(68,4 \%)$ et l'acétate de menthyle (16,9\%). Les autres composés représentatifs identifiés dans les HE sont: la menthone $(5,0 \%)$, l'isomenthone $(2,4 \%)$, le menthofurane $(2,3 \%)$ et la pipéritone $(1,5 \%)$.

\section{Activité antioxydante}

La mesure de l'activité antioxydante a donné les résultats représentés sur la Figure 1. Des pourcentages d'inhibition de $38 \%, 57 \%$ et $73 \%$ ont été notés pour les HE de M. arvensis respectivement aux concentrations de 10, 25 et $50 \mathrm{mg} / \mathrm{ml}$. La $\mathrm{CI}_{50}$ qui représente la concentration nécessaire pour inhiber 50\% des radicaux de DPPH est de $19,5 \mathrm{mg} / \mathrm{ml}$. Pour le trolox, les pourcentages d'inhibition sont de $10 \%, 67 \%, 84 \%$ et $85 \%$ respectivement pour les concentrations de $10^{-3} ; 5.10^{-3} ; 10^{-2}$ et $10^{-1}$ $\mathrm{mg} / \mathrm{ml}$.

\section{Activité insecticide}

La Figure 2 montre l'évolution de la mortalité des insectes en fonction de la concentration d'HE et de la durée du traitement. Le témoin blanc T0 et celui traité avec de l'acétone $\mathrm{T} 1$ enregistrent des mortalités quasi nulles. Néanmoins, pour l'acétone, la mortalité atteint $18 \%$ après 72 heures. Pour les lots traités avec des HE de $M$. arvensis, les mortalités augmentent avec la durée d'exposition. Ces mortalités sont de $10 \%, 10 \%, 30 \%$ et $14 \%$ après 24 heures respectivement pour T2, T3, T4 et T5. Au bout de 48 heures, $52 \%$ de mortalité sont notées dans le traitement $\mathrm{T} 2,92 \%$ pour $\mathrm{T} 3,76 \%$ pour T4 et $81 \%$ pour T5. Les mortalités après 72 heures sont de $98 \%$ pour $\mathrm{T} 2$ et $\mathrm{T} 3$ et deviennent maximales pour $\mathrm{T} 4$ et $\mathrm{T} 5$.

Tableau 2 : Composition chimique d'huiles essentielles de Mentha arvensis L. du Sénégal.

\begin{tabular}{lcc}
\hline Composés & $\begin{array}{c}\text { Indices de } \\
\text { rétention }\end{array}$ & $\begin{array}{c}\text { Composition } \\
(\mathbf{\%})\end{array}$ \\
\hline Octan-3-ol & 995 & 0,2 \\
Limonène & 1033 & 0,3 \\
1,8-Cinéole & 1037 & 0,1 \\
Linalol & 1100 & 0,1 \\
Menthone & 1162 & $\mathbf{5 , 0}$ \\
Menthofurane & 1172 & $\mathbf{2 , 3}$ \\
Isomenthone & 1175 & $\mathbf{2 , 4}$ \\
Menthol & 1184 & $\mathbf{6 8 , 4}$ \\
neo-Isomenthol & 1195 & 0,5 \\
cis-Carvéol & 1234 & 0,3 \\
Pulégone & 1246 & 0,1
\end{tabular}




\begin{tabular}{lcc} 
Pipéritone & 1262 & $\mathbf{1 , 5}$ \\
Acétate de néomenthyle & 1273 & 0,2 \\
iso-Pipériténone & 1277 & 0,3 \\
Acétate de menthyle & 1294 & $\mathbf{1 6 , 9}$ \\
$\beta$-Bourbonène & 1400 & 0,2 \\
cis-Jasmone & 1413 & 0,3 \\
$E$-(ß)-Caryophyllène & 1437 & 0,4 \\
$\beta$-Gurjunène & 1456 & 0,2 \\
Oxyde de caryophyllène & 1600 & 0,3 \\
\hline Monoterpènes oxygénés & & 98,3 \\
Monoterpènes hydrocarbonés & & 0,3 \\
Sesquiterpènes oxygénés & & 0,6 \\
Sesquiterpènes hydrocarbonés & & 0,8 \\
\hline
\end{tabular}
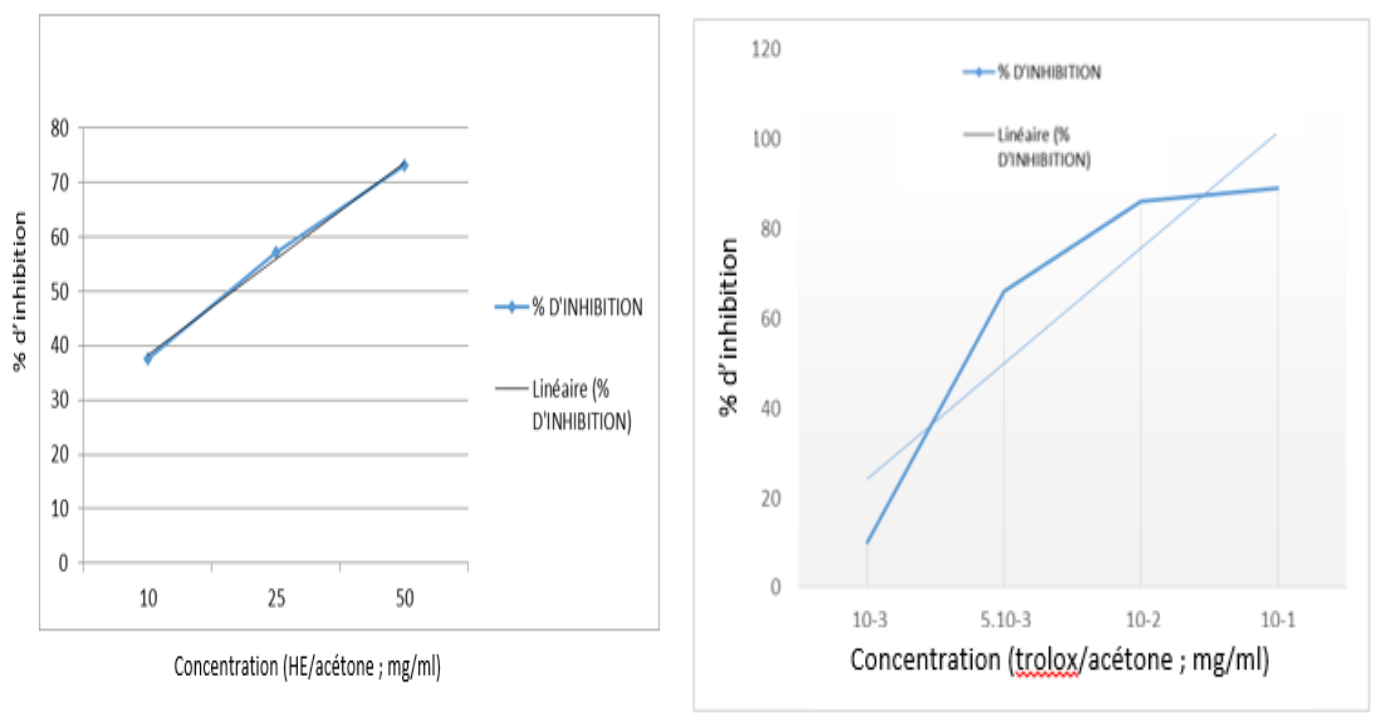

Figure 1: Evolution du pourcentage d'inhibition du radical DPPH en fonction de la concentration d'huiles essentielles de Mentha arvensis et du trolox. 


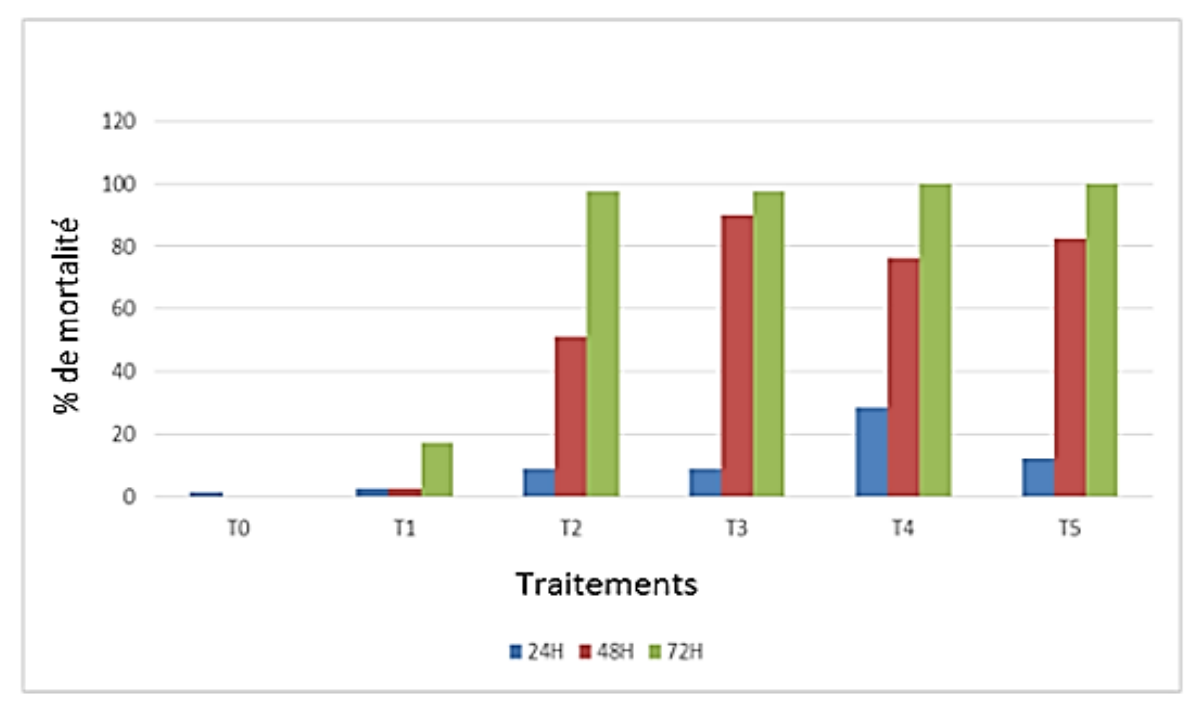

Figure 2 : Taux de mortalité des insectes en fonction du traitement.

\section{DISCUSSION}

La composition chimique obtenue dans les HE est typique de M. arvensis. En effet, les HE contiennent principalement du menthol à hauteur de 68,4\%. Ce dernier est un monoterpène qui est souvent identifié comme composé majoritaire dans plusieurs HE de $M$. arvensis. Verma et al. (2010) ont rapporté 61,9 à $82,2 \%$ de menthol dans les HE de $M$. arvensis tandis que Padalia et al. (2013) ont obtenu 73,7 à $85,8 \%$. Le menthol a une forte valeur ajouté ; c'est d'ailleurs le constituant le plus recherché dans les essences de menthes. Il est notamment utilisé dans la fabrication de sirops, de pastilles, de bonbons, etc.

Les HE étudiées présentent une capacité d'inhibition du radical DPPH. En d'autres termes, elles possèdent une activité antioxydante. Cette activité augmente avec la concentration mais reste faible par rapport au trolox. Cependant, comparée à l'activité d'autres HE, celle des HE de M. arvensis du Sénégal $\left(\mathrm{CI}_{50}=19,5 \mathrm{mg} / \mathrm{ml}\right)$ reste relativement intéressante. En effet, l'activité est forte par rapport à celle de Mentha pulegium d'Algérie $\left(\mathrm{CI}_{50}=29,4 \mathrm{mg} / \mathrm{ml}\right)$ et faible par rapport à celles de $M$. pulegium et de $M$. piperita du Maroc dont les $\mathrm{CI}_{50}$ sont respectivement de 4,8 et $6,4 \mathrm{mg} / \mathrm{ml}$ (Ou-Yahia, 2015 ; Achiri, 2018). Toutefois, le potentiel antioxydant peut varier selon la méthode utilisée. Dans la présente étude, l'activité antioxydante a été évaluée par la réduction du radical DPPH par la solution d'HE. Cette réduction est réalisée par transfert d'un hydrogène de la substance antioxydante vers le DPPH. L'activité antioxydante des HE de $M$. arvensis du Sénégal pourrait donc être due à la forte présence du menthol (2isopropyl-5-méthylcyclohexanol) qui possède un hydrogène actif dans sa structure. Cette capacité antioxydante rend les HE de $M$. arvensis plus intéressantes; elles pourraient notamment être utilisées dans la conservation des aliments contre le rancissement ou même dans un domaine, en l'occurrence la lutte contre le vieillissement des cellules humaines.

L'activité insecticide des HE testée contre S. zeamais a montré des mortalités croissantes avec la durée de traitement. Ces mortalités sont totales ou presque totales au bout de 72 heures. Cela fait que les HE de $M$. arvensis pourraient être utilisées en protection des denrées stockées contre les insectes ravageurs. Toutefois, il est à préciser que l'efficacité d'une HE contre un insecte est relative et dépend, entre autres, de l'insecte, du type de formulation et de la dose appliquée (Khani et Asghari, 2012; Bossou et al., 2015). De récents travaux ont montré l'importance du mode d'utilisation des HE. Dans ce domaine, les travaux de Guèye (2012) relatifs à la protection d'épis de maïs ont montré une 
efficacité totale en sept mois de stockage par utilisation de couches de plantes entières de Hyptis spicigera en alternance avec celles d'épis à l'intérieur des greniers. D'ailleurs, Kéita et al. (2001) proposent de réduire les plantes en poudre avant de les introduire dans les stocks. Ceci permettrait d'assurer une distribution plus uniforme des principes actifs afin de pouvoir protéger tous les grains.

\section{Conclusion}

Le présent travail a montré que les HE de Mentha arvensis du Sénégal contiennent principalement du menthol, composé à forte valeur ajoutée dans le domaine alimentaire. L'étude a permis d'observer un bon potentiel antioxydant dans les HE, ce qui pourrait être très intéressant dans la conservation des aliments. Ces HE causent aussi de fortes mortalités chez $S$. zeamais, principal ravageur du maïs. Elles pourraient donc constituer une alternative à l'utilisation des pesticides de synthèse qui, bien qu'efficaces, causent d'énormes dégâts sur l'environnement et la santé ; c'est une piste de valorisation des HE en biopesticides tout comme les fumigants spécialement dans la conservation des denrées. Dans des études ultérieures, il serait intéressant d'approfondir davantage les travaux sur cette plante et d'autres plantes médicinales afin d'isoler, de purifier et identifier les principes actifs responsables des activités biologiques.

\section{CONFLIT D'INTERETS}

Les auteurs déclarent qu'il n'y a aucun conflit d'intérêts.

\section{CONTRIBUTIONS DES AUTEURS}

SMD a réalisé le travail expérimental et rédigé l'article. MTG a orienté et supervisé les travaux. EHBN a participé aux analyses chromatographiques et l'exploitation des résultats. AT a participé aux tests antioxydants et l'exploitation des résultats. PSC a participé aux tests insecticides et l'exploitation des résultats. CHS a participé aux tests antioxydants et l'exploitation des résultats. M-LF a supervisé les analyses chromatographiques.

\section{REFERENCES}

Achiri R. 2018. Etude chimique et activité antioxydante des huiles essentielles de Mentha pulegium, Lavandula angustifolia et Verbena officinalis au niveau de la pépinière de Ain Fettouh. Mémoire de Master en Chimie, spécialité Chimie des Produits Naturels, Faculté des Sciences, Université de Tlemcen, 42 p.

Adams RP. 2001. Identification of Essential Oil Components by Gas Chromatography/Quadrupole Mass Spectrometry. Allured Publishing Co: Carol Stream IL- USA.

Bossou AD, Ahoussi E, Ruysbergh E, Adams A, Smagghe G, De Kimpe N, Avlessi F, Sohounhloue DCK, Mangelinckx S. 2015. Characterization of volatile compounds from three Cymbopogon species and Eucalyptus citriodora from Benin and their insecticidal activities against Tribolium castaneum. Industrial Crops and Products, 76: 306-317. DOI: http://dx.doi.org/10.1016/j.indcrop.2015. 06.031

Boukhebti H, Chaker AN, Belhadj H, Sahli F, Ramdhani M, Laouer H, Harzallah D. 2011. Chemical composition and antibacterial activity of Mentha pulegium L. and Mentha spicata L. essential oils. Scholars Research Library, 3(4): 267 275.

Cardiet G, Fuzeau B, Barreau C, FleuratLessard F. 2012. Contact and fumigant toxicity of some essential oil constituents against a grain insect pest Sitophilus oryzae and two fungi, Aspergillus westerdijkiae and Fusarium graminearum. Journal of Pest Science, 85: 351- 358. DOI: 10.1007/s10340-0110400-3

Cissokho PS, Guèye MT, Sow EH, Diarra K. 2015. Substances inertes et plantes à effet insecticide utilisées dans la lutte contre les insectes ravageurs des céréales et légumineuses au Sénégal et en Afrique de l'Ouest. International Journal of Biological and Chemical Sciences, 9(3): 1644-1653.

DOI: http://dx.doi.org/10.4314/ijbcs.v9i3.43 
Curutchet A, Dellacassa E, Ringuelet JA, Chaves AR, Viña SZ. 2014. Nutritional and sensory quality during refrigerated storage of fresh-cut mints (Mentha $x$ piperita and M. spicata). Food Chemistry, 143: 231-238. DOI: http://dx.doi.org/10.1016/j.foodchem.201 3.07.117

Dabiré C, Nébié RHC, Bélanger A, Naro M, Sib Sié F. 2011. Effet du séchage de la matière végétale sur la composition chimique de l'huile essentielle et l'activité antioxydante d'extraits de Ocimum basilicum L. International Journal of Biological and Chemical Sciences, $\quad \mathbf{5}(3)$ : $\quad 1082-1095$. DOI : $10.4314 /$ ijbcs.v5i3.72218

Dai DN, Thang TD, Emmanuel EE, Abdulkabir OO, Ogunwande IA. 2015. Study on essential oil of Mentha aquatica L. from Vietnam. American Journal of Essential Oils and Natural Products, 2(4):

12-16. https://www.essencejournal.com/archive s/2015/2/4/A

Diop SM, Guèye MT, Ndiaye I, Ndiaye EHB, Diop MB, Heuskin S, Fauconnier ML, Lognay G. 2016. Chemical composition of essential oils and floral waters of Mentha longifolia (L.) Huds. from Senegal. American Journal of Essential Oils and Natural Products, 4(1): 46-49. http://www.essencejournal.com/ vol4/issue 1/pdf/4-1-2.1.pdf.

Diop SM. 2018. Contribution à la valorisation des plantes aromatiques du Sénégal : étude des constituants chimiques d'huiles essentielles et d'eaux florales de basilic, citronnelle et menthes. Thèse de Doctorat en Chimie Moléculaire (option Organique), Faculté des Sciences et Techniques, Université Cheikh Anta Diop de Dakar, Sénégal, 183 p.

El-hawary SS, Taha KF, Monem ARA, Kirillos FN, Mohamed AA. 2013. Chemical composition and biological activities of peels and leaves essential oils of four cultivars of Citrus deliciosa var. tangarina. American Journal of Essential Oils and Natural Products, 1(2): 1-6. https://www.essencejournal.com/archive s/2013/1/2/A

Guèye MT, Goergen G, Ndiaye S, Asiedu EA, Wathelet JP, Lognay G, Seck D. 2013. Efficiency of traditional maize storage and control methods in rural grain granaries: a case study from Senegal. Tropicultura, 31(2): 39-46. DOI: https://www.academia.edu/16171670

Guèye MT, Lognay G, Seck D, Wathelet JP. 2011. Lutte contre les ravageurs des stocks de céréales et de légumineuses au Sénégal et en Afrique occidentale: synthèse bibliographique. Biotechnology, Agronomy, Society and Environment, 15(1): 183-194. https://www.researchgate.net/publication 150235215

Guèye MT. 2012. Gestion intégrée des ravageurs de céréales et de légumineuses stockées au Sénégal par l'utilisation de substances issues de plantes. Thèse de Doctorat en Sciences Agronomiques et Ingénierie Biologique, Gembloux Agro Bio-Tech, Université de Liège, Belgique, $216 \mathrm{p}$.

Ilboudo Z, Dabiré LCB, Nébié RCH, Dicko IO, Dugravot S, Cortesero AM, Sanon A. 2010. Biological activity and persistence of four essential oils towards the main pest of stored cowpeas, Callosobruchus maculatus (F.) (Coleoptera: Bruchidae). Journal of Stored Products Research, 46: 124-128.

DOI:

10.1016/j.jspr.2009.12.002

Joulain D, König WA. 1998. The Atlas of Sesquiterpene Data Hydrocarbons. E.B.Verlag: Hamburg- Germany.

Kéita SM, Vincent C, Schmita JP, Arnason JT, Bélanger A. 2001. Efficacy of essential oil of Ocimum basilicum L. and $O$. gratissimum $\mathrm{L}$. applied as an insecticidal fumigant and powder to control Callosobruchus maculatus (Fab.) [Coleoptera: Bruchidae]. Journal of Stored Products Research, 37: 339-349. DOI: 0022-474X/01/\$

Khani A, Asghari J. 2012. Insecticide activity of essential oils of Mentha longifolia, Pulicaria gnaphalodes and Achillea 
wilhelmsii against two stored product pests, the flour beetle, Tribolium castaneum, and the cowpea weevil, Callosobruchus maculatus. Journal of Insect Science, 12: $73 . \quad$ DOI: https://www.ncbi.nlm.nih.gov/pmc/articl es/PMC3593703/

Kwee EM, Niemeyer ED. 2011. Variations in phenolic composition and antioxidant properties among 15 basil (Ocimum basilicum L.) cultivars. Food Chemistry, 128: 1044-1050. DOI: 10.1016/j.foodchem.2011.04.011

Ncibi S, Attia S, Diop SM, Ammar M, Hance T. 2020. Bio-insecticidal activity of three essential oils against Rhyzopertha dominica (Fabricius, 1792) (Coleoptera: Bostrichidae). African Entomology, 28(2): 339-348. DOI: https://doi.org/10.4001/003.028.0339

Ngom S, Diop M, Mbengue M, Faye F, Kornprobst JM, Samb A. 2014. Composition chimique et propriétés antibactériennes des huiles essentielles d'Ocimum basilicum et d'Hyptis suaveolens (L.) Poit récoltés dans la région de Dakar au Sénégal. Afrique Science, 10(4): 109-117. DOI: https://www.ajol.info/index.php/afsci/iss ue/view/12404

Ngom S, Perez RC, Mbow MA, Fall R, Niassy S, Cosoveanu A, Diop SM, Ndiaye EHB, Diop M, Lognay G. 2018. Larvicidal activity of Neem oil and three plant essential oils from Senegal against Chrysodeixis chalcites (Esper, 1789). Asian Pacific Journal of Tropical Biomedicine, 8(1): 67-72. DOI: 10.4103/2221-1691.221140

Nguyen PM, Kwee EM, Niemeyer ED. 2010. Potassium rate alters the antioxidant capacity and phenolic concentration of basil (Ocimum basilicum L.) leaves. Food Chemistry, 123: 1235-1241. DOI: 10.1016/j.foodchem.2010.05.092
Ou-Yahia D. 2015. Activités antimicrobienne et antioxydante des huiles essentielles de : Mentha piperita et Mentha pulegium. Mémoire de Master en Sciences et Techniques : Biotechnologie Microbienne, Faculté des Sciences et Techniques, Université Sidi Mohammed Ben Abdellah, 45 p.

Padalia RC, Verma RS, Chauhan A, Sundaresan V, Chanotiya CS. 2013. Essential oil composition of sixteen elite cultivars of Mentha from western Himalayan region, India. Maejo International Journal of Science and Technology, 7(01): 83-93. DOI: 10.14456/mijst.2013.7

Rao BRR. 1999. Biomass and essential oil yields of cornmint (Mentha arvensis L. f. piperascens Malinvaud ex Holmes) planted in different months in semi-arid tropical climate. Industrial Crops and Products, 10: 107-113. DOI: https://doi.org/10.1016/S09266690(99)00012-6

Thiam A, Guèye MT, Sanghare CH, Ndiaye EHB, Diop SM, Cissokho PS, Diop MB, Ndiaye I, Fauconnier ML. 2020. Chemical Composition and Antiinflammatory Activity of Apium graveolens var. dulce Essential Oils from Senegal. American Journal of Food Science and Technology, 8(6): 226-232. DOI: 10.12691/ajfst-8-6-1

Verma RS, Rahman L, Verma RK, Chauhan A, Yadav AK, Singh A. 2010. Essential Oil Composition of Menthol Mint (Mentha arvensis) and Peppermint (Mentha piperita) Cultivars at Different Stages of Plant Growth from Kumaon Region of Western Himalaya. Journal of Medicinal and Aromatic Plants, 1(1): 13-18. https://scholar.google.co.in/citations?use $\mathrm{r}=3$ iC6NGwAAAAJ\&hl=en. 